\title{
ANALYZING THE ROAD NETWORK STRUCTURE OF TANG-TIBET ROAD AND BUILDING A SPATIAL INFORMATION SYSTEM FOR ITS TIBET SECTION
}

\author{
Mengjie CHI ${ }^{1,2}$, Yi LIU ${ }^{1}$ \\ ${ }^{1}$ Dept. of Urban planning, Southeast University, 2 Sipailou, Nanjing, Jiangsu, China - (230208987, 230208479)@seu. edu. cn \\ ${ }^{2}$ Faculty of Information Engineering, Xizang Minzu University, 6Wenhui Road, Xianyang, Shaanxi,China
}

Keywords: Tang-Tibet Road, Road Network Structure, Space Information System, Preservation and Development

\begin{abstract}
:
Since the Tang Dynasty (618-907 AD), the Tang-Tibet Road has been the only way from inland China to Qinghai and Tibet, and even to other countries such as Nepal and India. It ties and bonds various ethnic groups and regions, integrates cultural memories and cross-cultural communication achievements from ancient times to the present, and witnesses the dynamic propagation of the culture. Affected by the environment, climate, and wars, Tang-Tibet Road was often impossible to travel on or through intermittently during its progressive development in history. Routes and lines of each of its sections changed from time to time; eventually, an ancient road network was formed, consisting of one trunk road, two subsidiary roads in the north and south, several branches, and scattered auxiliary routes separated from the system, among which there were both outward-oriented international passages and inward-oriented passages. Nonetheless, research on Tang-Tibet Road is insufficient at the current stage. Regarding the problems summarized based on the review of the research situation, the present work probes deeper into the network structure of Tang-Tibet Road. How historical corridor is generated and evolved is understood from a regional perspective. In particular, strategies to design a space information system for the Tibet section of Tang-Tibet Road are explained to promote the exploration and use of cultural heritages in Tibet, in an effort to preserve these heritages while developing Tibet's society and economy.
\end{abstract}

A significant trend of current world heritage preservation is the protection of cultural routes. Most of these routes have evolved and changed remarkably during the long-term development in history. In practice, some of their sections are completely different from what they used to be under the acts of urbanization progression and natural calamities, making it challenging to define them accurately. In many cases, a large amount of multidisciplinary research is required to support such a definition.

Tang-Tibet Road is very famous in ancient Chinese history. It has been the only way from inland China to Qinghai and Tibet, and even to other countries such as Nepal and India since the Tang Dynasty (618-907 AD). Tang-Tibet Road started from Chang' an (Xi' an, Shaanxi today), the capital of Tang Dynasty, and eventually reached RaSa (Lhasa, Tibet today), the capital of Tubo Kingdom, spanning five Chinese provinces (Shaanxi, Gansu, Qinghai, Sichuan, and Tibet) and totaling a length of 3,000 kilometers. Crossing the roof of the world, it traverses Western China and connects the Qinghai-Tibet Plateau with inland China in terms of politics, economy, and culture; it also links the twin cities and countries to Southwestern China, playing a pivotal role in maintaining China's territorial integrity and safeguarding the national unity. Tang-Tibet Road served as a carrier for different cultures to communicate and exchange and acted as a support for cross-regional conversations; thus, its significance was self-evident. With the rapid urbanization progress, the preservation of cultural heritages and the joint development of society and economy constitute the new requirements of the times, calling for a further investigation on how to preserve and use Tang-Tibet Road in modern times.

\section{RESEARCH SITUATION AND PROBLEMS OF TANG-TIBET ROAD}

\subsection{Tibetan Plateau not included in systems related to the Silk Road}

The world-famous Silk Road served as a hub of political, economic, and cultural exchanges between China and other countries in ancient times. In particular, it could be divided into the land Silk Road and the marine Silk Road. The former refers to the land passage across the Eurasia continent through Hexi Corridor started in Chang'an (Xi'an, Shaanxi today) that Zhang Qian (200-114 BC) opened in the Western Han Dynasty (206 $\mathrm{BC}-24 \mathrm{AD}$ ); the latter is considered the marine trade route connecting the Southeast Asia, West Asia, Europe, and Africa started in Guangdong and Fujian. However, routes to the Tibetan Plateau are excluded from this international trade network (Huo, 2021a) (Figure 1). In the past, understandings of routes connecting the outside world and Tibetan Plateau were entirely based on documents filed after the establishment of Tubo Kingdom in the seventh century. Situations of transportation on the Tibetan Plateau in the early days were rarely introduced in ancient documents. Now, many scholars have engaged in Tibetan research. Relics and archaeological discoveries of various periods prove that the route between this plateau and the outside world has long existed since prehistoric times. The relationship between the Tibetan Plateau and the "Silk Road" has gradually attracted much attention - Tibetan Plateau has contributed to the establishment of the Silk Road network in history.

The most well-known ancient Tibetan road is the Tang-Tibet Road. It was a route for communications between Tibet and inland China, the West, and Central and South Asia since the seventh century when Tubo regime was established. Many Chinese ancient documents, such as The New Book of Tang (a work of official Tang history), The Old Book of Tang (the first classic historical work about the Tang dynasty), Tongdian (a Chinese institutional history and encyclopedia text), and Full Tang Literature (a collection of articles in Tang Dynasty, including those in the Five Dynasties after Tang vanished) have left records for this road. Because the route from Chang'an to 
Shanzhou - the east section of the Tang-Tibet Road's trunk - basically overlaps with the Silk Road's south line, it is also referred to as the Southern Silk Road. Thus, records of this section are pretty rich. Post stations and directions of the west section that connects Shancheng County and RaSa, the capital of Tubo Kingdom, are merely introduced in both Chinese and Tibetan literature due to its harsh environment. The extreme cold weather and tall mountains and vast rivers that blocked most of the lanes on this section resulted in desolated settlements that were far from the inland, creating a huge barrier for the successors to probe into the history of this section, with few systematic studies.

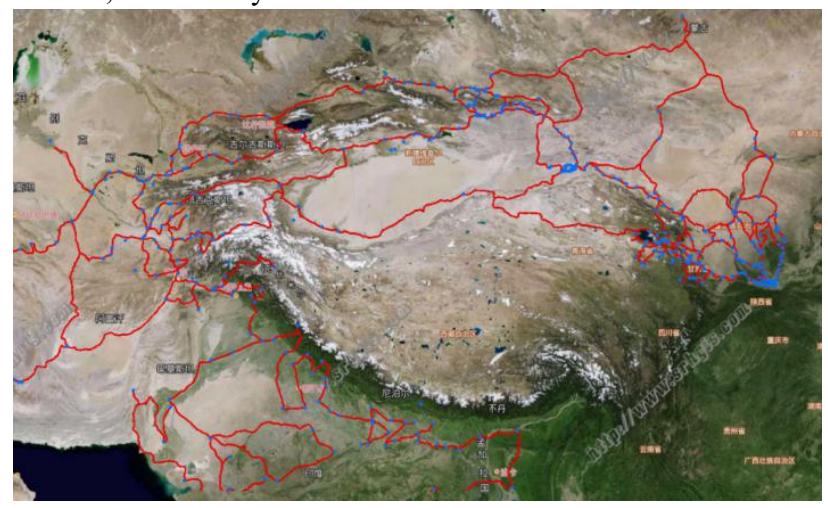

Figure 1. Routes to the Tibetan Plateau are excluded from Routes of the Silk Road (Tang Dynasty) (source: Silk Road Historical Geography Information Open Platform)

1.2 Lots of linear research on Tang-Tibet Road, with less emphasis on its system networking and time dynamism

Tibet has complex terrain and greatly-changing topography, in which the relatively undulating and gentle mountains, wide valleys, and basins are distributed in opposite directions; lakes are widespread, with the periglacial landform. Climate changes in this region are diverse, and extreme weather often occurs. Affected by the environment, climate, and wars, Tang-Tibet Road was shaped progressively and was often impossible to travel on or through intermittently during its development. Routes and lines of each of its sections changed from time to time; eventually, an ancient road network was formed, consisting of one trunk road, two subsidiary roads in the north and south, several branches, and scattered auxiliary routes separated from the system, among which there were both outward-oriented international passages and inward-oriented passages connecting inland China and the Tibetan Plateau with outside world based on different demands during different periods. Tang-Tibet Road has been formed and evolved for at least 1,300 years. According to the transit time and route, its history of evolution can be classified into three stages: early, middle, and late, but this classification is slightly different from the middle and late stages of Tubo's history (Yu et al., 2018).

The present-available historical data and contemporary files about the routes of Tang-Tibet Road are mostly written records; the few illustrations are only demonstrations to the trunk road, without any marks of the subsidiary roads and branches, nor are these illustrations corresponded to the real topography. For this reason, map translation, map drawing, and accurate positioning in the Geographic Information System (GIS) are required.

\subsection{Inadequate research on the relationship between Tibet's historic corridor and historical towns}

In different times, people are provided with different route choices based on different purposes, which explains how a virtual-real transportation network is formed. Any changes in this network can merely affect the formation of intersections of the main lines and routes; that is, network nodes where passengers are dense-populated will always develop into human settlements and towns. During the formation of historic corridors and towns, various ethnic groups have interacted and blended for a long time, forming a historical pattern of harmonious and multi-cultural coexistence and co-prosperity. Historical cities, as nodes of communication corridors, experience fewer changes in location, where population gatherings and activities are frequent, leaving rich historical records. The existing material cultural heritages, such as grottoes, temples, ancient towns, ancient streets, inns, and post stations, strongly support the existence and direction of the historic corridor. Therefore, research on the historic corridor is inseparable from that on historical cities and towns. The clear direction of ancient routes can be found quickly and accurately from the perspective of the cultural corridor nodes, thus forming a network system. In addition, the research on integrating historic corridors and historical cities and towns in Tibet provides theoretical and practical significances for consolidating the sense of community of the Chinese nation, promoting exchanges and blending of various ethnic groups, and carrying out ethnic works.

\subsection{Insufficient efforts made to preserve Tibetan history and culture}

Due to Tibet's high altitude, harsh climate, stratum change, and sparse population, there are fewer traces of remains on the historic corridor. What is worse, the preservation work is facing the dual-pressure of natural changes and human destruction. At present, most of Tibet's cultural heritages are preserved and researched through the "point approach" (measures are made on the levels of buildings, ruins, and remains). There are few plans for the general layout of lines, planes, and regional protection and use. While preserving and developing historical cities and towns simultaneously, it is bound to encounter the contradictory of unitary preservation and solitary development.

China has a wealth of linear cultural heritages. Constructing heritage corridors is not only the need for preserving the various linear cultural heritages but also the demand for developing efficient and forward-looking ecological infrastructure in the context of rapid urbanization. Historic corridors are the development trends of regional and integrated cultural heritage preservation. The coordinated integration of Tibetan cultural heritage corridors and historical cities can contribute to the overall and coordinated preservation of precious cultural heritages. While shaping the regional characteristics, it also brings benefits to various livelihood undertakings, such as the development of urbanization and the construction of new agricultural villages.

\section{ROAD NETWORK STRUCTURE RECONSTRUCTION OF TANG-TIBET ROAD}

Tang-Tibet Road connects regimes on inland China and Tubo Kingdom in the Middle Ages in history. The composition and changes of its routes reveal the political, military, and cultural exchanges between Tang and Tubo. The trunk road that originated in Chang'an, passed through Shancheng County, and reached Lhasa can be outlined based on literature. Moreover, two subsidiary roads in the north and south can be marked as per the contemporary archaeological discoveries and historical documents. In addition to various branches, Tang-Tibet Road 
can be restored and reconstructed generally, acting as the material that verifies and supplements the history.

\subsection{The origin and historical environment of Tang-Tibet Road}

At the beginning of the seventh century, King Songtsen Gampo (604-649 AD) conquered Sumpa, Zhangzhung, and some scattered Tibetan tribes. Eventually, he unified the Tibetan Plateau and established a powerful slavery regime --the Tubo Kingdom. In its early days, Tubo Kingdom stretched to ancient Nepal in the south, Monpa-Lhoba region that located in southeastern Tibet in the east, and Zhangzhung in the west; it connected to the West with Kunlun Mountains; its northeast neighbor was the Tuyuhun regime that ruled ancient Qinghai, with Tanggula Mountains acting as the natural barrier. The territory of Tubo Kingdom was roughly equivalent to that of the Tibet Autonomous Region today. Tubo Kingdom expanded its surrounding lands by force for nearly two hundred years after its establishment, helping the once loose tribal union grow into a plateau dynasty with an extremely large area and unprecedented power. The continuous expansion of territories also exerted a profound impact on the historical process of inland dynasties and even countries of South and Central Asia. In the seventh century when the Tubo Kingdom was just established, there were four competing neighboring political forces: the Tang Dynasty to the east, the Umayyad Caliphate of Tazi to the west, the Turkut tribes to the north, and Sindhu to the south. $708 \mathrm{AD}$ witnessed the failure of the alliance between Tubo Kingdom and Hindu Shahi destroyed by the Arabians, forcing Tubo to give up casting its greedy eyes on the lands under Arabian Empire's reign. Since then, Tubo started to turn its sights to the east. Its east neighbor, the Tang Dynasty, ruled vast lands and had a huge population, were known for its long history and prosperous civilization. Tang's warm weather and fabulous wealth were exuding fatal attraction undoubtedly. Inevitably, eastward expansion became the strategic goal of Tubo Kingdom. From a historical point of view, the expansion wars started by Tubo have objectively promoted the development and progress of transportation between Tibetan Plateau and its surrounding areas.

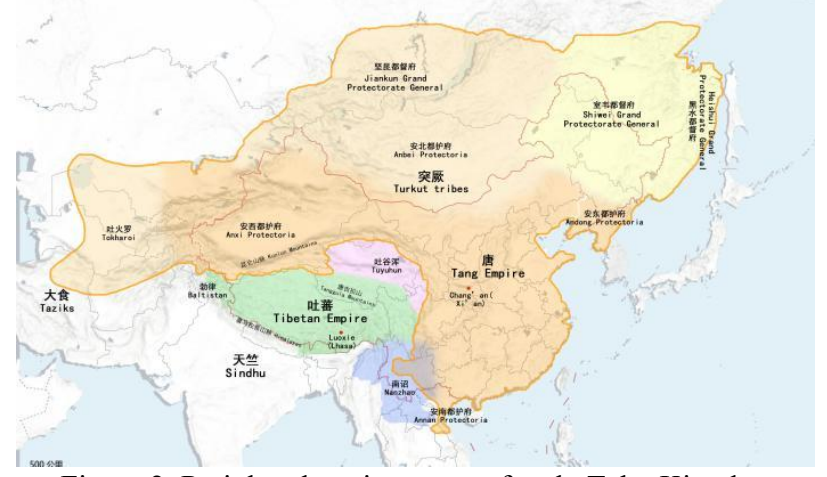

Figure 2. Peripheral environment of early Tubo Kingdom

The Hehuang Valley on the Tang-Tibet Road was the junction station between inland China and Qinghai-Tibet Plateau in ancient times. A smooth route had stood between Hehuang and Tibet since Qin (221-207 BC) and Han (206 BC - 220 AD) Dynasties; its history could even trace back to the Neolithic period 6,000 years ago, laying a foundation for the formation and development of Tang-Tibet Road. All the emperors of Han and Tang Dynasties made efforts to run the Hehuang Valley. A county was set up here, and garrison troops and peasants were send here to open up wasteland and grow food grain and safeguard the borders at war; these measurements, on the one hand, ensured the unimpeded transportation between ancient China and the West, promoted the political, economic, and cultural exchanges between inland China and the Hehuang region, and developed the transportation in this region. On the other hand, after Songtsen Gampo unified the Qinghai-Tibet Plateau and established the regime, the relationship between Tubo and the Tang dynasty continued to develop. Both sides kept sending ambassadors to each other, which created a situation that was described as "Between Tang and Tubo, precious goods are traded, and the smooth route has welcomed loads of guests from afar" (Dong, 1988). This undoubtedly promoted the final formation of this ancient transportation road, with a history of more than 1,300 years till now.

\subsection{Road network structure of Tang-Tibet Road}

Tang-Tibet Road has been formed and perfected gradually with the Tang-Tibet's political, economic, military, and cultural exchanges and the military expansion of Tubo Kingdom. It varied in different historical periods. After more than 200 years, an ancient Tang-Tibet road network consisting of a trunk road, two subsidiary roads, and several branches is finally formed.

Trends of the whole Tang-Tibet Road were poorly documented. The New Book of Tang (Ou et al., 1975) and The Old Book of Tang (Liu, 1975) recorded the routes from Chang'an (Xi'an, Shaanxi today) to RaSa (Lhasa today) that Princess Wencheng (628-680 AD) and Princess Jincheng (698-739 AD) took, who came to Tubo to marry the Tubo Kings; also, these two books also had words about Tang-Tibet ambassadors' trips between Tang and Tubo. According to these records, the Tang-Tibet Road was roughly divided into a east and a west section. The east section lay in the territory of Tang Dynasty, approximately from Chang'an (Xi'an today) to Shancheng County (Xining, Qinghai today). The west section, by contrast, accounted for two thirds of the entire trunk, stretching from Shancheng County (Xining today) to RaSa (Lhasa today), all within the territory of Tubo Kingdom (Figure 3).

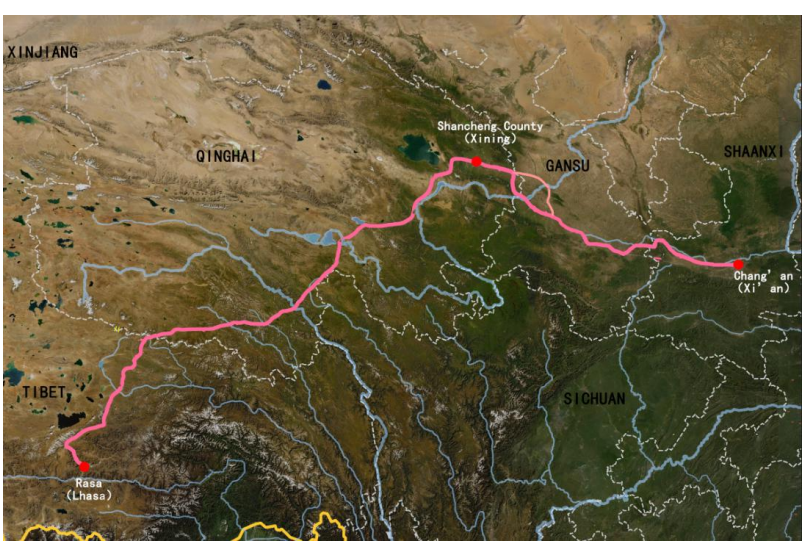

Figure 3. Tang-Tibet Road's trunk

The trunk road was the "official road" documented in literature, with post stations and other facilities along the way. In historical records --Annals of Yuanhe County written by Li Jifu (758-814 AD) of Tang Dynasty, for example -- "heading the west after leaving Chang'an, passing Longzhou, Qinzhou, Weizhou, Wuzhou, Lanzhou, and Hezhou, one will finally arrive Shanzhou (Li, 2011)," namely, the east section of Tang-Tibet Road's trunk. The approximate route was: Chang'an (Xi'an today) --Fengxiang (Fengxiang County, Shaanxi today) --Longzhou (Long County, Shaanxi today) --Qinzhou (Tianshui, Gansu today) --Weizhou (Longxi County 
today) --Linzhou (Lintao County today) --Lanzhou (Lanzhou today)/Hezhou (Linxia today) --Shanzhou (Xining today).

Overlapping with the Silk Road to a large extent, Tang-Tibet Road can be regarded as a part of it. According to the historical materials, this road was already the official road with a history to learn from in the Sui Dynasty (581-618 AD) at the latest, linking the central government with the prefectures and counties in the Longyou region. Since the Tang Dynasty, many post stations were set up along the route from the Guanzhong Plain to Hehuang Valley in order to ensure the smooth traffic flow between the capital and Hehuang region. In the meantime, prefectures and counties were established, connecting towns and post stations on the road to form the official road from Chang'an to Shancheng County in the Tang Dynasty.

Ancient Tang documents suggested multiple line choices for Tang ambassadors to visit Tubo Kingdom. According to Full Tang Literature, for instance, Lv Wen (771-811 AD) started out from Qingshui County and arrived in Tubo via ancient Hezhou City. Tubo Preach of The New Book of Tang wrote that Liu Yuanding entered the land of Tubo Kingdom via Wuzhou, Lanzhou, and Qinghai. Thus, there were two lines to reach ancient Shanzhou City on the east section of Tang-Tibet Road's trunk: the Hezhou line and the Lanzhou line. Tang-Tibet ambassadors left traces on both lines during their round trips. Based on existing documents, most of them took the Hezhou line. There were fewer records of the Lanzhou line in that it was nearly a hundred li (50 kilometers) farther than the Hezhou line(Dao, 2011); meanwhile, the former provided worse geographical conditions than the latter. Except for fortresses such as the Yellow River and the Mantianling Ridge, the topography of the Hezhou line was mostly plain and flat Huangshui Valley and valley lanes. Roads stretching from the west of Lanzhou to Shanzhou, by contrast, were dangerous mountains and gorges (such as Honggupo and Laoya Canyon), which was difficult to pass through. That was why ancient ambassadors usually took the Hezhou line to travel between Tang and Tubo. The Bingling Temple Annotation found in Bingling Temple Grotto and a Tibetan history book The Political and Religious History of Anduo (mdo-smad-chos-vbyung) recorded that while Princess Wencheng leaving for Tubo, she stayed Hezhou for a short period and ordered to engrave figures of the Buddha, revealing the significance of the Hezhou line.

Most routes on the east section of Tang-Tibet Road were located in the densely populated agricultural areas in the Tang Dynasty; thus, the roads were flat, and the official post stations were dense, leaving quite abundant and clear records of post stations along this section. Thus, the situation will not be repeated here.

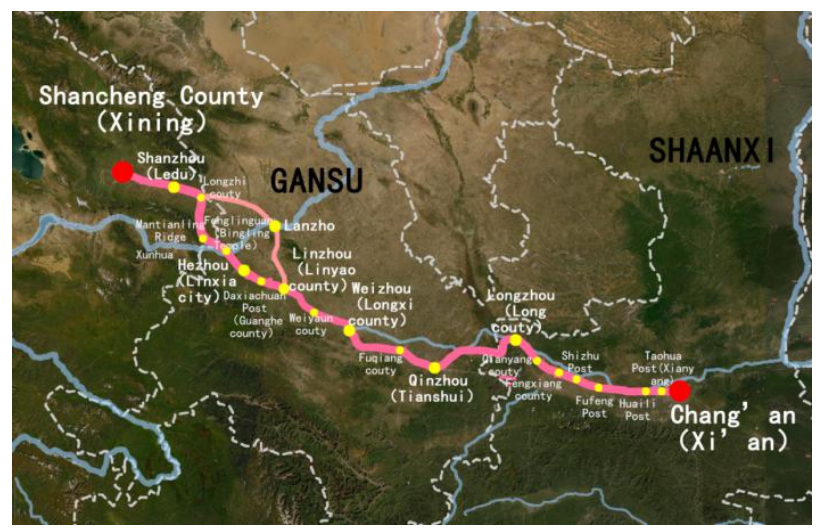

Figure 4. The east section of Tang-Tibet Road
The west section of Tang-Tibet Road referred to the journey from Shancheng County to RaSa, the capital of Tubo Kingdom. The approximate route was Shancheng County (Xining today) --Chiling (Riyue Mountain today) --Dafei River (Gonghe County today) --Zhonglong Post --(Chindu County today) --Jiezhiqiao (Yushu today) --Yema Post (Nyainrong County today) --Gechuan Post (Nagqu County today) --Nongge Post (Yangbajain, Lhasa today) --RaSa (Lhasa today). This section, by contrast, is poorly documented due to the tall mountains and vast rivers that blocked most of its lanes and the extreme cold weather caused by the high altitude, resulting in desolated settlements that are far from the inland, creating a huge barrier for the successors to probe into its history.

The New Book of Tang · Geography Records · Shancheng County provided records specially for the routes and directions of the west section (Ou et al., 1975), in which the names for places and ethnic groups were almost the same. Once these geographical coordinates are determined, the approximate route of this ancient road from the west of Shancheng County to RaSa can be outlined, roughly divided into the following five major subsections:

The first subsection started from Shancheng County to Chiling, namely the passage from Ledu, Qinghai, to Riyue Mountain on the southeastern corner of Qinghai Lake passing Xining today. Vital stations and towns it passed included the Linbo City, the Dingrong City, the Stone Fort City, Baishuijun, and the Suirong City.

The second subsection headed from Chiling via Dafei River (Gonghe County) to the ferry crossing on the north bank of the Yellow River. In particular, it passed Nalu Post (Daheba Township, Xinghai County today), Nuanquan Post (Daheba Township, Xinghai County today), Liemohai (Kabahai, also known as the Bitter Sea), the Qieji Prairie centered on the Shazhuyu River Basin (Dafei River) , and the north bank of the Yellow River (the bank of Yellow River governed by Madoi County today). Some important ruins are distributed along this subsection, such as the memorial halls for General Wang Xiaojie (?-697 AD) and Princess Mushan (?). People traveling between Tang and Tubo at that time took the same route as they take now.

The third subsection spanned from the ferry crossing of the Yellow River to Zhonglong Post (Chindu County today) passing through Bohai (Ngoring Lake today). Passing through the Yellow river ferry crossing, one would arrive at Bayan Har Mountains immediately. Zhonglong Post was 470 li (235 kilometers) away from the Yellow River.

The fourth subsection denoted to the miles from Yushu, Qinghai to Tibet, namely the "Tibet Route" in history. Crossing the Xiyue River (Grand Zhaqu River today) from Zhonglong Post, one would arrive at the western border of ancient Duomi Nation (Batang Prairie today). Then, after passing the $\mathrm{Bu} \mathrm{Ha}$ Chi (Buha River), Lie Post (Jielong Township, Yushu County today), Jiezhiqiao (Geishabiandi, Yushu County today), Po Post (Zhaxilawu Temple, Yushu today), the bridge of Dayue River (Zhaqu today), Xinuoluo Post (west of Zadoi County today), people would reach Tanggula Mountains. Satou Hisashi and Wu Jing'ao once debated the route and direction of this subsection in Yushu due to the different coordinates of Ziqu River since the records were too old to be remain consistent.

The fifth section lay in the Tibet Autonomous Region today, entering the the valley floor of Lhasa via the pass of Tanggula Mountains and Changtang. The Zihemang Post (the West Sog County today) was the place where the princess received Tang ambassadors visiting Tubo. Passing through Yema Post, (Nyainrong County today), Kentian of Tubo Kingdom (Baixiong Township, Nyainrong County today), Gechuan Post 
(Nagqu County today), Habulan Post (Sangxiong Township, Nagqu County today), Tuliji Post (the north of Sangqu Bridge today), Tangluoyeyi Mountains (Nyenchen Tanglha Mountains today), Zanpu Alter (the south foot of Nyenchen Tanglha Mountains today), and Nongge Post (Yangbajain, Lhasa today), travelers finally reached the destination - RaSa (Lhasa today), the capital of Tubo Kingdom (Chen, 1989).

The section from Shancheng County to RaSa could be further divided into a south trunk and a north trunk. These trunks were not official roads; instead, they were closely connected to the eastward expansion of Tubo Kingdom, without any Tang post stations. The north trunk spanned from Shanzhou (Ledu today) to Shazhou (Dunhuang today), including two branches: (1) Shanzhou (Ledu today) --Biandukou of Guangzhou (Minle County, Zhangye today) and Sunan Yugur Autonomous County --Suzhou (Jiuquan today) --Guazhou (Jiuquan today) --Shazhou (Dunhuang today); (2) Shanzhou (Xining today) --Chiling (Riyue Mountain today) --Fuqi, capital of Tuyuhun --Dulan --Shazhou (Dunhuang today) .

The south trunk spanned from Yushu via eastern Tibet to Lhasa The approximate route was: Yushu --Sêrxü County, Luhuo County, and Dawu County, Sichuan --Zhag'yab County and Markam County, Chamdo Prefecture --Gongbo'gyamda County --Nyingchi Prefecture --Lhasa. This trunk included three branches: (1) RaSa (Lhasa today) --Tuyuhun or Tangut (borders of Gansu, Qinghai, and Sichuan) --Bailan (Yushu/Tibetan Autonomous Prefecture of Golog, Qinghai, Ngawa Tibetan and Qiang Autonomous Prefecture, Sichuan) --Songzhou (Songpan County, Sichuan); (2) RaSa (Lhasa) -"six sgang of mdo-khams" (Chamdo Prefecture in the Tibet Autonomous Region) --Jiezhiqiao (Yushu, Qinghai) --"Deng" (Sêrxü County, Sichuan) --Luhuo (Luhuo County, Sichuan) --Daofucheng Fort (Daofu, Sichuan); (3) RaSa (Lhasa) --Nyingchi Prefecture --Gongbo'gyamda County --Zhag'yab County and Markam County, Chamdo Prefecture --Gyamda --Sêrxü County, Sichuan --Yushu, Qinghai.

The first two branches are eastward military expansion routes of Tubo Kingdom recorded in both Tibetan an Chinese. At present, only graves and tombs can validate their existence. The third branch is proved to be the propagation route of Buddhism to Tubo Kingdom, along which there are archaeological sites such as the Buddhism ruins, cliff inscriptions, and graves (Qiao et al.,2015a). This branch is never mentioned in literature, which is a newly discovered branch determined through archaeology (Yu et al., 2018).

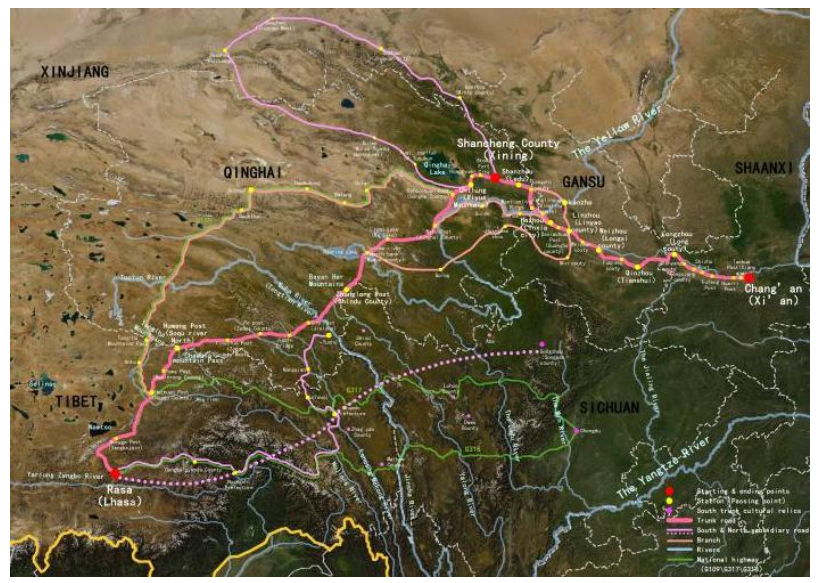

Figure 5. Trunk road, subsidiary road and branch of Tang-Tibet Road

To sum up, routes from Tubo Kingdom to other regions were opened or formed in different periods. The increasingly convenient transportation development laid the solid foundation for not only the political unity within the Tibetan Plateau --the establishment of Tubo Kingdom --but also the Kingdom's external expansion. Trunks and branches of Tang-Tibet Road connected the plateau and the inland, linked the West with the northwestern and the southwest regions of ancient China, and provided a transportation route to Central and South Asia, leaving its marks in the history of transportation in China and foreign countries.

\section{BUILDING THE SPACE INFORMATION SYSTEM FOR THE TIBET SECTION OF TANG-TIBET ROAD}

Tibet is vast and sparsely populated. The diversity and complexity of its cultural heritages result in tedious workloads of heritage preservation and management. Compared with inland China, Tibet subjects to plateau technical restraints; in addition, traditional management methods cannot play their expected roles under the unique geographical conditions. Thus, a complete data information system shall be established to integrate and use information about Tibet's historical cultural heritage, which has a strong macroscopic orientation and is helpful to the precise governance and improvement of the efficiency of heritage preservation and management.

\subsection{Starting from the point --Precise heritage preservation based on 3D technology}

Part of the Lhasa Jokhang Temple caught on fire in 2018; in the same year, the National Library of Brazil also suffered from fire hazard. In 2019, the world-famous Notre-Dame de Paris was burned down. A series of incidents have sounded the alarm for the safety protection works of cultural artifacts around the world. While emphasizing the traditional safety protection measures, the urgency of digital protection for cultural artifacts cannot be ignored. The Dunhuang Academy can serve as an example; it has been exploring digital protection approaches since 1993. Among the 30 grottoes in Dunhuang, 4,430 square meters of murals from ten dynasties are displayed in the "Digital Dunhuang" project. China has issued a series of policies successively in recent years, such as Opinions on Continuing Strengthening Cultural Artifact Works, Three-Year Plan for "Internet + Chinese Civilization," and Opinions on Implementing the Project for the Preservation and Use of Revolutionary Cultural Artifacts (2018-2022), putting forward clear requirements for the digital protection of heritages.

Since the Potala Palace was listed as a World Cultural Heritage in December 1994, the central and local governments of China have attached great importance to the preservation of cultural artifacts in Tibet. The Potala Palace Fine Surveying and Mapping Project was launched on June 11th, 2016 --the Chinese Cultural Heritage Day. This was the first large-scale high-precision digital surveying and mapping project in Tibet.

Buildings of the Potala Palace are of complex structures and magnificent scales. Hence, the surveying and mapping team adopted various modern technologies, including 3D laser scanning, multi-view image reconstruction, and Unmanned Aerial Vehicles (UAVs)-based aerial photography, to collect high-precision 3D information and high-definition textures of the buildings and the surrounding terrain. The collected information will play its role in establishing the Potala Palace monitoring and control network. It satisfies people's needs for the protection, management, utilization, and research of the Potala Palace and lays a technical foundation for the comprehensive preservation of cultural artifacts. As digital heritages increase, the importance of building a management system based on the Internet of Things (IoT) and big data 
becomes increasingly prominent, exerting a significant impact on increasing the degree of data refinement, improving data storage and output performances, optimizing heritage management procedures, and improving propagation and displaying approaches.

Cultural artifacts in high-altitude regions often remain less affected by human activities due to the sparse population. However, global warming, frequent extreme weather, and active geological structures bring severe survival threats to many corridor heritages in China due to the lack of fundamental protection facilities. Confined to a single item, the traditional "point-based" preservation approach is inadequate and costly, failing to show the linkage effect. The digital surveying and mapping project in Tibet calls for in depth development and a subtle balance between the quantity and quality.

\subsection{Oriented to the line --Preservation and utilization of cultural routes based on GIS}

In the digital age, there are already mature GIS applications in preserving and managing urban township architectural heritages and historical cities, towns, and villages. Every link of information collection, resource digitization, data management, data analysis, preservation and early warning, and public participation of heritages can be governed effectively.

Cultural heritages in Qinghai-Tibet Plateau are rich in types, including cultural artifacts, historic sites, ruins, buildings, historical towns and centers, heritage routes, and cultural landscapes, with a wide variety and a wide range of resources. Nonetheless, various systems are insufficiently linked, management standards are quite different, and data feedback often lags behind. In this case, it seems particular necessary to establish a GIS-based management information system for urban cultural heritages on the basis of sorting out all elements of the cultural heritage to be protected through urban geographic information data and GIS spatial analysis technology.

First, data integration should be the starting point to preserve and use cultural routes in Tibet. The accurate routes can be determined based on the current research results of the ancient Tang-Tibet Road. A standardized 2D space can be formed using the existing urban geospatial information to serve for the subsequent tasks, such as the input, storage, and query of various historical and cultural resource data. Second, from the perspective of cultural heritage documentation, all the elements of the list of cultural routes and cultural heritages should be sorted out and determined. Each item should be registered, reviewed, and supplemented in a hierarchical and categorical manner. Function of viewing various information should be designed, including the preservation status and description information, warning levels, scene photos, surrounding environment and preservation status of the cultural heritage related to the Tang-Tibet Road. Third, from the perspective of line-based preservation, buffer zones should be designed for each heritage element (points and lines) according to the assessed value, thereby assisting in delimiting the boundaries of the preservation area. Control contents, including the core preservation area, the construction control area, and the environmental coordination area, should act as the basis for the subsequent city and township planning, thereby revitalizing the historical and cultural resources while ensuring the healthy development of townships and cities along the road. 3.3 Integrated as per the plans --Plans to preserve the
regional historic corridor based on the big data platform

Based on the "point-based" and "line-based" works described above, GIS service engine, big data, and cloud computing technology are practiced to build an open platform for scientific research and data management services with network as its central framework. The historical and cultural spaces of the Tibet Autonomous Region, as well as the construction of the future digital city platform, can be optimized in terms of coordination mechanisms and planning systems.

Regarding the coordination mechanism, the big data platform shall be built based on the four levels of infrastructure, data resources, application support, and display services to achieve the network collaboration and sharing of open data. With functions such as spatio-temporal framework, map library, map sharing, and data analysis, this platform can assist in multidisciplinary research and large-scale geoscience studies, thus improving the teaching and research capabilities and contributing to the disciplinary development. This platform can also achieve the information sharing and collaborative management of various departments, support the internal business functions of the management departments and the works of planning and design agencies, strengthen the communication between information management, status feedback, and action decisions in urban development and cultural heritage protection, and coordinate the development of various tasks. These functions can promote the efficient, dynamic, and comprehensive preservation and management of the city's cultural heritages, which is conducive to the revitalization of cultural heritages (He et al., 2018a).

Regarding the planning system, heritage resource attributes, spatial data, and various planning and project data, including the material cultural heritage entities of "point-line-plane" and the intangible heritages, should be integrated to build a systematic and comprehensive big data platform. The development status around the trunk roads should be assessed qualitatively and quantitatively based on the control lines, providing a basis for the management and revitalization strategies of cultural heritages (He, 2015b). A "multi-level and all-element cultural heritage protection system' can be established to promote the comprehensive preservation, integrated organization, intensive management, and overall development following the strategy of putting equivalent weights on preservation and development of cultural heritages led by the government with multiple participants.

Eventually, it is recommended to accelerate the construction of high-end think tanks, integrate the wisdom of the government, enterprises, and experts, and create an action-oriented, interdisciplinary, and professional think tank to address the problems and put forward suggestions and solutions to the challenges encountered during heritage preservation and socio-economic development of historical towns in Qinghai-Tibet Plateau.

\section{SUMMARY}

In October 2019, President Xi Jinping of China visited India and Nepal successively, during which he provoked the discussion on the possibility and perspectiveness of "Bangladesh - China - India - Myanmar" cooperation and delivered his expectations of China-Nepal railway across Himalayas. This illustrates the vigor of Tang-Tibet Road in a new historical period --the significance for the exchanges and communication between East and South Asia. The radiation effect of "Bangladesh - China - India - Myanmar Economic 
Corridor" shall drive the joint development of the three major economic sectors: South Asia, Southeast Asia, and East Asia, accelerating the development of Qinghai-Tibet region and its surrounding economies. In addition, China plans to hold a "Conversation on Asian Cultural Heritage Protection" in October 2021. These moves all demonstrate that the concept of "development with preservation and preservation with development" is getting deepened. Various fields of economic and social development are strengthening their intersection and interaction with cultural heritage preservation. As the core area and geographic highland of Asia, Qinghai-Tibet Plateau plays a vital role in cultural exchanges. The Tang-Tibet Road serves as a communication channel between China and West, Central, and South Asia. With the Belt and Road Initiative, the ancient road can open a new chapter of Tibet. Qinghai-Tibet Plateau is expected to become a thoroughfare to enter the world economic and cultural exchange system by combining Tang-Tibet Road and the Belt and Road.

\section{REFERENCES}

Chen Xiaoping, 1989. Tang-Tibet Road. Sanqin Publishing House, Xi'an, pp.1-196.

Dao xuan, 2011. Local records of Sakya. Shanghai Classics Publishing House, Beijing, pp.10-86.

Dong Hao et al., 1988. Full Tang Literature. Zhong Hua Book Company, Beijing, pp.226.

He Jing, 2015b. Research on the protective evaluation model of historical streets in Changsha city based on GIS.Hunan University.pp.6-8.

He Shao-ying et al., 2018a. A research on the construction of urban historical and cultural heritage management information system based on GIS. Journal of Guangdong University of Technology,35(05), pp. 38-44.

Huo Wei, 2021a. On the joint creation of "Silk road of the plateau"by ancient people of all ethnic groups on the QinghaiTibet Plateau. Journal of Minzu University of China,Vol.48, pp. $5-13$.

Li Jifu, 2011. Annals of Yuanhe County.National Library Press, Beijing, pp.1-187.

Liu Xu, 1975. The Old Book of Tang. Zhong Hua Book Company, Beijing, pp.68.

Ou Yangxiu et al., 1975. The New Book of Tang. Zhong Hua Book Company, Beijing, pp.1-21.

Qiao Hong et al.,2015a. A new chapter of prehistoric culture and Tubo cultural Archaeology in the three river source area of Yushu, Qinghai:The cultural remains of Tubo Period,4(24).

Yu Xiaohong et al., 2018. The structure of Tang-Tibet Road and Archaeological investigation and Research of cultural artifact. Zhongshan University Press, Guangzhou, pp.165. 